\title{
DERIVATIVE ESTIMATES FROM DISCONTINUOUS REALIZATIONS: SMOOTHING TECHNIQUES
}

\author{
Paul Glasserman \\ Room 3K-324 \\ AT\&T Bell Laboratories \\ Holmdel, NJ 07733
}

\author{
Wei-Bo Gong \\ Electrical and Computer Engincering \\ University of Massachusetts \\ Amherst, MA 01003
}

\begin{abstract}
We develop two methods for estimating derivatives of expectations from simulation of functions whose realizations are discontinuous in the parameter of differentiation. We take as motivating example the estimation of the sensitivity of expected terminal reward for processes on discrete state spaces. Both our methods use conditional expectations to smooth discontinuites. The first smooths the dependence on the differentiation parameter, while the second smooths dependence on the time parameter. The methods are illustrated through examples, including stochastic networks, networks of queues, and Markov processes.
\end{abstract}

\section{INTRODUCTION}

This paper considers the problem of estimating derivatives of expectations from realizations that are discontinuous functions of the parameter of differentiation. It is known that in its simplest form - the method of infinitesimal perturbation analysis (IPA) can only be applied in cases where the realizations are continuous in the parameter (see [4] for a discussion of the role of continuity in IPA). Here, we develop two modifications of IPA that "smooth" discontinuities in order to obtain unbiased derivative: estimates.

To motivate the problem of discontinuities, let $Z_{t}(\theta)$ be a parametric family of processes on a discrete state space $S$. For $f$ a real-valued function on $\mathbf{S}$, and fixerl $t_{f}>0$ define the terminal reward

$$
R(\theta)=f\left(Z_{t_{j}}(\theta)\right)
$$

Depending on what $Z_{\ell}$ and $f$ represent, $\mathrm{E}[R(\theta)]$ could be, for example, a mean queue length at $t_{f}$, or the probability that some system is still functioning at $t_{f}$. Suppose we want to estimate $d \mathrm{E}[R(\theta)] / d \theta$. The IPA estimate is $R^{\prime}(\theta)=d R(\theta) / d \theta$. But since $Z_{t}(\theta)$ takes only discrete values, the same is true of $R(\theta)$; hence $R^{\prime}(\theta)=0$ wherever it exists. As a function of $\theta, R$ is (at best) piecewise constant. The "sample derivative" $R^{\prime}(\theta)$ contains no information about the dependence of $\mathrm{E}[R(\theta)]$ on $\theta$.

This situation should be contrasted with that of cumulative reward given by

$$
L(\theta)=\int_{0}^{t_{f}} g\left(Z_{t}(\theta)\right) d t .
$$

Since $L$ takes on a continuum of values, it cannot be a priori declared discontinuous the way $R$ can. In fact, a variety of conditions on $Z_{t}(\theta)$ ensure that $\mathbf{E}\left[L^{\prime}(\theta)\right]=\mathbf{E}[L(\theta)]^{\prime}$; some are considered in [4],[5] and [6].

To deal with (1) and similar problems we consider two smoothing techniques. Both use conditional expectations, but in different ways. The first is an application of smoothed perturbation analysis (SPA) as developed in Gong and Ho [8] and Glasserman and Gong [ 7$]$. Roughly speaking, SPA uses an estimate of the form $\mathbf{E}[R(\theta) \mid \xi]^{\prime}$ where $\xi$ is determined by $\left\{Z_{t}(\theta), t \geq 0\right\}$. The condition $\xi$ is chosen so that $\mathbf{E}[R(\theta+h) \mid \xi]$ is continuous in $h$. We apply SPA to (1), after considering simpler problems. Our second approach to smoothing uses Dynkin's formula to convert (1) to (2) and then applies IPA. While the two methods are, in general, distinct, we identify a small class of problems for which both yicld the same estimator.

Previous work on calculating $d \mathrm{E}[R(\theta)] / d \theta$ (when $Z_{t}(\theta)$ is Markov) includes the numerical method in Heidelterger and Goyal [9], and the numerical and simulation methods considered in Fox [2]. (See also other references in [2].) These papers partly motivated our work. Our approach based on Dynkin's formula has close ties to the elegant compensator method introduced (earlier, independently) by Zazanis [11] via the single-server queue.

\section{SPA: THE BASIC IDEA}

Let $\gamma(\theta)$ be a random, piecewise constant function taking only the values $x_{1}, \ldots, x_{m}$. (Think of $\gamma(\theta)$ as the outcome of a simulation conducted at the parameter value $\theta$, in a situation where re-running the simulation using common random numbers with $\theta$ slightly perturbed may not change the value of $\gamma$.) If $\mathrm{E}[\gamma(\theta)]$ is differentiable, then

$$
\begin{aligned}
\frac{d \mathbf{E}[\hat{\gamma}(\theta)]}{d \theta} & =\lim _{h \downarrow 0} \mathbf{E}\left[\frac{\gamma(\theta+h)-\gamma(\theta)}{h}\right] \\
& =\lim _{h \downarrow 0} \mathbf{E}\left[\frac{\mathbf{E}[\gamma(\theta+h)-\gamma(\theta) \mid \gamma(\theta)]}{h}\right] .
\end{aligned}
$$

If the limit and the outer expectation can be interchanged, this becomes

$$
=\mathbf{E}\left[\lim _{h \mid 0} \frac{\mathbf{E}[\gamma(\theta+h)-\gamma(\theta) \mid \gamma(\theta)]}{h}\right]
$$




$$
=\mathbf{E}\left[\lim _{h \backslash 0} \sum_{\jmath=1}^{m} \frac{1}{h} P\left(\gamma(\theta+h)=x_{\jmath} \mid \gamma(\theta)\right)\left[x_{\jmath}-\gamma(\theta)\right]\right] .
$$

If we define

$$
q\left(x_{1}, x_{\jmath}\right)=\lim _{h 10} \frac{1}{h} P\left(\gamma(\theta+h)=x_{\jmath} \mid \gamma(\theta)=x_{1}\right),
$$

then

$$
\sum_{j=1}^{m} q\left(\gamma(\theta), x_{\jmath}\right)\left[x_{\jmath}-\gamma(\theta)\right]
$$

is an unbiased estimate of $\mathbf{E}[\gamma(\theta)]^{\prime}$. Bringing the limit fully inside the expectation in (3) (instead of only partially) would yield the uninteresting estimate $\gamma^{\prime}(\theta) \equiv 0$.

The estimate in (5) requires calculation of $q\left(x_{2}, x_{3}\right)$ (whereas $\gamma(\theta)$ is generated from simulation) which can be difficult. Evaluation of the limit in (4) is generally facilitated by conditioning on more information. Suppose, then, that $\xi=\xi(\theta)$ represents additional information available through simulation (including $\gamma(\theta)$ - i.e., $\gamma \in \sigma(\xi)$ ), and let

$$
Q\left(x_{j} ; \xi\right)=\lim _{h \downarrow 0} \frac{1}{h} P\left(\gamma(\theta+h)=x_{\jmath} \mid \xi\right) .
$$

Proceeding as in (3)-(5), this yields the estimate

$$
\sum_{j=1}^{m} Q\left(x_{j} ; \xi\right)\left[x_{3}-\gamma(\theta)\right]
$$

We think of $Q(x, ; \xi)$ as the (conditional) jump rate from $\gamma(\theta)$ to $x_{3}$, and $\left[x_{j}-\gamma_{i}(\theta)\right]$ as the effect or size of such a jump. Note that $Q(x, ; \xi)$ is, indeed, a conditional intensity for viewed as a stochastic process in $\theta$.

Taking this one step further, we can condition different jumps on different information. For $j=1, \ldots m$ let $\gamma \in \sigma\left(\xi_{3}\right)$. Writing

$\mathbf{E}[\gamma(\theta+h)-\gamma(\theta)]=\mathbf{E}\left[\sum_{\jmath=1}^{m} P\left(\gamma(\theta+h)=x_{\jmath} \mid \xi_{\jmath}\right)\left[x_{\jmath}-\gamma_{\gamma}(\theta)\right]\right]$ leads to the estimate

$$
\sum_{j=1}^{m} Q\left(x_{j} ; \xi_{\jmath}\right)\left[x_{j}--\gamma(\theta)\right]
$$

Tail, ring $\xi$, can make each $Q(x, \xi \xi)$ easier to calculate than $Q(x, ; \xi)$ with $\xi$ fixed. We will provide explicit expressions for $Q(\cdot ; \cdot)$ for a variety of examples.

As a trivial but illustrative example, consider the problem of estimating

$$
\frac{d}{d \theta} P(X(\theta)<t)=\partial_{\theta} F(t, \theta)
$$

when the dependence of $X$ on $\theta$ is via

$$
X(\theta)=X(1) / \theta
$$

If we set $\gamma(\theta)=\mathbf{1}\left\{X^{\prime}(\theta)<t\right\}$ then $P(X(\theta)<t)=\mathbf{E}[\gamma(\theta)]$.

We condition on $\gamma(\theta)$. Since $X$ is decreasing in $\theta$, when $\gamma(\theta)=1$ no increase in $\theta$ can change the value of $\gamma$; hence, whrn $\gamma(\theta)=1$ the estimate is zero. When $\gamma(\theta)=0$, we need to differentiate the rate at which it jumps to one:

$$
\begin{aligned}
\lim _{h \downarrow 0} & \frac{1}{h} P(\gamma(\theta+h)=1 \mid \gamma(\theta)=0) \\
= & \lim _{h \downarrow 0} \frac{1}{h} P(X(\theta+h)<t \mid X(\theta)>t) \\
= & \lim _{h \downarrow 0} \frac{1}{h} P\left(X(\theta)<\frac{\theta+h}{\theta} t \mid X(\theta)>t\right) \\
= & \lim _{h \downarrow 0} \frac{1}{h} \frac{F\left(\frac{\theta+h}{\theta} t, \theta\right)-F(t, \theta)}{1-F(t, \theta)} \\
= & \frac{f(t, \theta)}{1-F(t, \theta)} \frac{t}{\theta},
\end{aligned}
$$

where $f$ is the density of $F$. The relation (9) implics that $d X / d \theta=-X / \theta$, so this could be expressed as

$$
\frac{f(t, \theta)}{1-F(i, \theta)}\left(-\frac{d X}{d \theta}\right)_{x=t}
$$

(the second factor is the derivative of $X$ evaluated at $X=t$ ). This jump rate has a simple interpretation which extends to more general cases: The first factor is the "probability" that $X$ is infinitesimally greater than $t$, given that it is greater than $t$; when multiplied by $-d X / d \theta$ (evaluated at $X=t$ ) this yields the rate at which $I$ and $t$ change order.

To obtain the full estimate, we note that this order change can only occur when $X(\theta)>t$, and when it occurs,, jumps from 0 to 1 , yielding an effect of $[1-0]=+1$. Thus, we get

$$
1\{X(\theta)>t\} \frac{f(t, \theta)}{1-F(t, \theta)}\left(-\frac{d X}{d \theta}\right)_{X=t}[+1] .
$$

Furthermore, noting that (9) implies $F(x, \theta)=F(x \theta, 1)$, differentiation shows that $X$ admits the inversion representation

$$
\frac{d X}{d \theta}=-\frac{\partial_{\theta} F(X, \theta)}{\partial_{x} F(X, \theta)}
$$

Substituting (11) into (10) and taking the expectation we get

$$
(1-F(t, \theta)) \frac{f(t, \theta)}{1-F(t, \theta)}\left(\frac{\partial_{\theta} F(t, \theta)}{f(t, \theta)}\right)=\partial_{\theta} F(t, \theta)
$$

which is, of course, what we wanted.

Consider, next, a modification of the problem above in which we try to estimate 


$$
\frac{d}{d \theta} P\left(X_{1}(\theta)<X_{2}(\theta)\right)
$$

Let $i_{1}$ and $i_{2}$ be, respectively, the indices of the smaller and the larger of the $X_{1}(\theta)$. We condition on $\xi=\left(i_{1}, X_{i_{1}}\right)$ and consider the rate at which $X_{1}$ and $X_{2}$ change oriter:

$$
\lim _{h \backslash 0} \frac{1}{h} P\left(X_{1_{1}}(\theta+h)>X_{1_{2}}(\theta+h) \mid i_{1}, X_{i_{1}}\right) .
$$

Suppose there are functions $D_{\mathfrak{t}}, i=1,2$ such that

$$
\frac{d X_{\mathfrak{t}}(\theta)}{d \theta}=D_{\mathfrak{t}}\left(X_{\mathfrak{t}}(\theta), \theta\right)
$$

(more on this condition below). Formally, proceeding as above, we obtain the estimate

$\frac{f_{i_{2}}\left(X_{i_{1}}(\theta)\right)}{1-F_{i_{2}}\left(X_{i_{1}}(\theta)\right)}\left[\frac{d X_{i_{1}}}{d \theta}-D_{1_{2}}\left(X_{i_{1}}\right)\right]^{+}\left[1\left\{i_{1}=2\right\}-1\left\{i_{1}=1\right\}\right]$.

Since $D_{i_{2}}\left(X_{i_{1}}\right)$ has the interpretation

$$
D_{t_{2}}\left(X_{i_{1}}\right)=\left(\frac{d X_{i_{2}}}{d \theta}\right)_{x_{i_{2}}=X_{i_{1}}}
$$

the $[\cdot]^{+}$term is the rate at which the $X_{2}$ change order, given that $X_{t_{2}}$ is just greater than $X_{1_{1}}$. The "+" arises from the fact that as $h \mid 0$, the conditional probability in (12) is zero unless $X_{t_{1}}$ is increasing faster than $X_{t_{2}}$. Justification of this limit requires some elaboration on the dependence of the $X_{1}$ on $\theta$. This will be carried out in the next section.

\section{A CLASS OF NETWORK PROBLEMS}

We now extend the examples of the previous section to some functions of the form

$$
\gamma(\theta)=\Gamma\left(X_{1}(\theta), \ldots, X_{n}(\theta)\right)
$$

where $\Gamma$ maps $\mathrm{R}^{n}$ to a finite sot, and the $X_{1}(\theta)$ are independent. The situation we have in mind is one in which $\Gamma$ represents a network property and the $X$, are stochastic capacities, arc lengths or lifetimes; but this interpretation is not essential. Let $i_{1}, \ldots, i_{n}$ be order indices for the $X_{2}$ :

$$
X_{i_{1}} \leq X_{i_{2}} \leq \ldots \leq X_{i_{n}}
$$

We restrict attention to $\Gamma$ determined by a function $g$ via

$$
\Gamma\left(X_{1}, \ldots, X_{n}\right)=g\left(i_{1}, \ldots, i_{n}\right)
$$

As an example, take the $X$, to be component lifetimes in a system that functions so long as at least one component in each of a collection of fixed subsets $C$, functions. Then if $g$ is the indicator of the event

$$
X_{k}=\min _{j} \max _{i \in C_{j}} X_{t}
$$

$\mathrm{E}[\gamma]$ is the probability that the system ultimately fails due to failure of component $k$. Other types of $\Gamma$ can be handled using sinilar techniques.

We begin by taking up in more detail the calculation of $Q(\cdot ; \cdot)$, imposing the following provisional conditions:

(A1'). Each $X_{1}$ is, with probability one, a differentiable function of $\theta$.

(A2 ${ }^{\circ}$ ). Each $X_{2}(\theta)$ has a density $f_{\imath}(\cdot, \theta)$ (with cdf $F_{\imath}(\cdot, \theta)$ ).

For some of our calculations we will need a strengthening of (A1') which generalizes (9). Fix a nominal value of $\theta$ and suppose there is a function $\Phi$, such that

$$
\Phi_{\imath}\left(X_{\imath}(\theta), h\right)=X_{\imath}(\theta+h)
$$

Think of $\Phi_{2}$ as the general solution to the differential equation specified by (13). Note that $\Phi_{\imath}(x, 0)=x$, and

$$
\begin{aligned}
\partial_{h} \Phi_{\imath}(x, 0) & =\lim _{h \downarrow 0} \frac{\Phi_{\imath}(x, h)-\Phi_{2}(x, 0)}{h} \\
& =D_{\imath}(x)=\left(\frac{d X_{2}}{d \theta}\right)_{X_{1}=x}
\end{aligned}
$$

Also, if $\Phi_{2}$ has an inverse (with respect to its second argument), then (17) shows that it is given by $\Phi_{t}^{-1}(\cdot, h)=$ $\Phi_{1}(\cdot,-h)$.

We impose

(A1). For each $X_{2}$ there is a $\Phi$, satisfying (17) which is continuously differentiable in both arguments and strictly monotonic in the second argument throughout a neighborhoort of $\theta$.

Though somewhat unintuitive, (A1) is fairly general, especially when (A1') is known to hold. (A candidate $\Phi$ is provided by the solution to (11).) To show how (A1) is used, we return to (12). We can rewrite it as

$$
\begin{aligned}
& \lim _{h \backslash 0} \frac{1}{h} P\left(\Phi_{1_{1}}\left(X_{i_{1}}(\theta), h\right)>\Phi_{1_{2}}\left(X_{i_{2}}, h\right) \mid i_{1}, X_{1_{1}}\right) \\
= & \lim _{h \backslash 0} \frac{1}{h} P\left(X_{1_{2}}<\Phi_{1_{2}}\left(\Phi_{i_{1}}\left(X_{1_{1}}, h\right),-h\right) \mid i_{1}, X_{1_{1}}\right) \\
= & \lim _{h ! 0} \frac{1}{h} \frac{F_{2_{2}}\left(\Phi_{2_{2}}\left(\Phi_{i_{1}}\left(X_{i_{1}}, h\right),-h\right)\right)-F_{1_{2}}\left(X_{1_{1}}\right)}{1-F_{1_{2}}\left(X_{1_{1}}\right)},
\end{aligned}
$$

provided

$$
\Phi_{2_{2}}\left(\Phi_{1_{1}}\left(X_{1_{1}}(\theta), h\right),-h\right)>X_{1_{1}}(\theta)
$$


and zero otherwise. Unless (19) holds for all sufficiently small $h>0$, the limit is zero. When (19) does hold for all small $h$, the chain rule yields

$$
\frac{f_{i_{2}}\left(X_{1_{1}}\right)}{1-F_{i_{2}}\left(X_{i_{1}}\right)} \cdot \frac{d}{d h}\left[\Phi_{i_{2}}\left(\Phi_{i_{1}}\left(X_{i_{1}}, h\right),-h\right)\right]_{h=0} .
$$

Note that $h$ appears in both arguments of $\Phi_{1_{2}}$. The derivative can be expressed as

$$
\begin{gathered}
\lim _{h 10} \frac{1}{h}\left(\Phi_{1_{2}}\left(\Phi_{\imath_{1}}\left(X_{\imath_{1}}, h\right),-h\right)-\Phi_{\imath_{2}}\left(\Phi_{1_{1}}\left(X_{\imath_{1}}, 0\right),-h\right)+\right. \\
\left.\Phi_{\imath_{2}}\left(\Phi_{\imath_{1}}\left(X_{\imath_{1}}, 0\right),-h\right)-\Phi_{\imath_{2}}\left(\Phi_{\imath_{1}}\left(X_{\imath_{1}}, 0\right), 0\right)\right)
\end{gathered}
$$

since the middle terms cancel. If $\Phi_{1}$ and $\Phi_{2}$ are $C^{1}$, we can take the limit as $h \downarrow 0$ separately for $h$ in the first and second arguments of $\Phi_{12}$ to get

$$
\begin{aligned}
& \lim _{h \downarrow 0} \frac{\Phi_{i_{2}}\left(\Phi_{i_{1}}\left(X_{i_{1}}, h\right), 0\right)-\Phi_{i_{2}}\left(\Phi_{i_{1}}\left(X_{i_{1}}, 0\right), 0\right)}{h} \\
+ & \lim _{h \downarrow 0} \frac{\Phi_{i_{2}}\left(\Phi_{i_{1}}\left(X_{i_{1}}, 0\right),-h\right)-\Phi_{i_{2}}\left(\Phi_{i_{1}}\left(X_{i_{1}}, 0\right), 0\right)}{h} .
\end{aligned}
$$

Since $\Phi_{i_{2}}(x, 0)=x=\Phi_{i_{1}}(x, 0)$, this is

$$
\begin{aligned}
& \lim _{h 10} \frac{\Phi_{i_{1}}\left(X_{i_{1}}, h\right)-\Phi_{1_{1}}\left(X_{i_{1}}, 0\right)}{h} \\
+ & \lim _{h 10} \frac{\Phi_{1_{2}}\left(X_{i_{1}},-h\right)-\Phi_{i_{2}}\left(X_{i_{1}}, 0\right)}{h} .
\end{aligned}
$$

Equation (18) now shows that this is

$$
\frac{d X_{\imath_{1}}}{d \theta}-D_{t_{2}}\left(X_{\imath_{1}}\right)
$$

Combining this with the case where (19) does not hold for all small $h$ yields (14).

We now apply similar ideas to $\gamma$ as defined via $\Gamma$ and $g$ in (15) and (16). Since $g$ could, in general, take up to $n$ ! different values, derivation of an SPA estimate for $d \mathbf{E}[\gamma(\theta)] / d \theta$ would seem to require consideration of $n !-1$ possible changes in $\gamma$. Fortunately, most of the changes occur with negligible probability as $h \downarrow 0$. Let $\underline{i}=\left(i_{t}, \ldots, i_{n}\right)$ be the sequence of order indices, and let $\underline{i}$ j be the sequence obtained from $\underline{i}$ by transposing $i_{j}$ and $i_{j+1}$. Let $\mathcal{A}(\theta)$ be the set consisting of $\underline{i}(\theta)$ and all $\underline{i}_{j}(\theta), j=1, \ldots, n-1$. Then (A1') and (A2') imply that

$$
P(\underline{i}(\theta+h) \notin \mathcal{A}(\theta))=o(h) ;
$$

thus, we only nced to consider the $n-1$ possible order changes of consecutive $X_{2}$.

For each of these possible order changes we need to find the conditional jump rate, as in (6). For the jump from $\underline{i}$ to $i_{j}$ we condition on $\xi_{j}=\left(\underline{i}, X_{i j}\right)$. The general form of the estimate (8) is then given by

$$
\sum_{\jmath=1}^{n-1} Q\left(\underline{i}_{j} ; \xi_{\jmath}\right)\left[g\left(\underline{i}_{\jmath}\right)-g(\underline{i})\right]
$$

(We have abbreviated $Q\left(g\left(\underline{i}_{j}\right) ; \xi_{\jmath}\right)$ to $Q\left(\underline{i}_{j} ; \xi_{\jmath}\right)$ ) As in the example of the previous section, each jump rate takes the form

$$
Q\left(\underline{i}_{j} ; \xi_{\jmath}\right)=\hat{f}_{\jmath}(0)\left[\frac{d X_{i_{j}}}{d \theta}-D_{i_{\jmath}+1}\left(X_{i_{\jmath}}\right)\right]^{+},
$$

where $\hat{f}_{j}$ is the conditional density given $\xi_{j}$ of the residual lifetime of $X_{i+1}$. This density is given, up to proportionality, by

$$
\hat{f}_{\jmath}(t) \sim f_{\imath_{\jmath}+1}\left(X_{\imath \jmath}+t\right) \prod_{k=\jmath+1}^{n}\left(1-F_{i_{k}}\left(X_{\imath_{\jmath}}+t\right)\right) .
$$

Equality is obtained by dividing the right side by its integral (over $0 \leq t<\infty$ ) to make it a probability density. Determination of this type of normalization constant is the one non-trivial step in implementing SPA estimates.

We prove unbiasedness of this estimator under two additional conditions, the first a strengthening of (A2'):

(A2). For each $i$ and $\theta$ there is a $\lambda_{i}(\theta)$ such that for all $x \geq 0, f_{2}(x, \theta) /\left(1-F_{i}(x, \theta)\right) \leq \lambda_{2}(\theta)$.

(A3). There is a constant $B>0$ such for every $i$, with probability one, $\left|d X_{2} / d \theta\right| \leq B\left(X_{2}+1\right)$.

Integration shows that (A3) implies

$$
\left|X_{\imath}(\theta+h)-X_{\imath}(\theta)\right| \leq\left(\epsilon^{B h}-1\right)\left(X_{2}(\theta)+1\right) .
$$

Proposition 1. Suppose (A1)-(A3) hold, and suppose that every $X$, has a finite expectation. Then, if $\mathbf{E}[\gamma(\theta)]$ is differentiable,

$$
\mathbf{E}\left[\sum_{j=1}^{n-1} Q\left(\underline{i}_{\jmath} ; \xi_{\jmath}\right)\left[g\left(\underline{i}_{\jmath}\right)-g(\underline{i})\right]\right]=\frac{d \mathbf{E}[\gamma(\theta)]}{d \theta}
$$

Proof. There are two parts to the proof: showing that our expression for $Q\left(\underline{i}_{j} ; \xi_{3}\right)$ is correct, and justifying the interchange of the limit and the expectation in

$$
\begin{aligned}
\lim _{h \mid 0} \frac{1}{h} \mathbf{E} & {\left[\sum_{\jmath=1}^{n-1} P\left(\underline{i}(\theta+h)=\underline{i}_{\jmath} \mid \xi_{\jmath}\right)\left[g\left(\underline{i}_{\jmath}\right)-g(\underline{i})\right]\right]=} \\
\mathbf{E} & {\left[\lim _{h \backslash 0} \frac{1}{h} \sum_{j=1}^{n-1} P\left(\underline{i}(\theta+h)=\underline{i}_{\jmath} \mid \xi_{\jmath}\right)\left[g\left(\underline{i}_{\jmath}\right)-g(\underline{i})\right]\right] . }
\end{aligned}
$$

The left side is the derivative of $\mathrm{E}[\gamma(\theta)]$; the right side is the expectation of the estimator. Verification that $Q\left(\underline{i} ; \xi_{3}\right)$ 
is correct is similar to the example carried out above su we omit it. For the interchange, we bound the probability of a jump:

$$
\begin{aligned}
& \frac{1}{h} P\left(\underline{i}(\theta+h)=\underline{i}_{\jmath} \mid \xi_{\jmath}\right) \\
& \leq \frac{1}{h} P\left(X_{i},(\theta+h)>X_{\imath_{3+1}}(\theta+h) \mid \xi_{\jmath}\right) \\
& \leq \frac{1}{h}\left|\frac{F_{i_{3}+1}\left(\Phi_{i,}\left(\Phi_{i_{3+1}}\left(X_{2}, h\right),-h\right)\right)-F_{i_{3+1}}\left(X_{i \jmath}\right)}{1-F_{i_{3}+1}\left(X_{i}\right)}\right| \text {. }
\end{aligned}
$$

Applying the mean value theorem and (A2), this is

$$
\leq \lambda_{i, 1+1}\left|\frac{\left.\Phi_{i,}\left(\Phi_{i_{1+1}}\left(X_{i,}, h\right),-h\right)\right)-X_{i,}}{h}\right| \text {. }
$$

The numerator is bounded by

$\left|\Phi_{1,}\left(\Phi_{\imath \jmath+1}\left(X_{1}, h\right),-h\right)-\Phi_{2 \jmath+1}\left(X_{\imath}, h\right)\right|+\left|\Phi_{\imath \jmath+1}\left(X_{2}, h\right)-X_{\imath \jmath}\right|$

Using (21) for each term and simplifying, (22) becomes

$$
\leq \lambda_{\imath_{3+1}} \frac{1}{h}\left(\epsilon^{2 B h}-1\right)\left(X_{\imath_{3}}+1\right) \text {. }
$$

Another application of the mean value theorem yields

$$
\leq \lambda_{\imath+1}\left(2 B h e^{2 B h}\right)\left(X_{\imath}+1\right) .
$$

Since $\mathrm{E}\left[X_{1}\right]<\cdots$, the dominated convergence theorem now justifies the interchange.

\section{TERMINAL REWARD IN QUEUEING NETWORKS}

We return, now, to the problem described in the introduction, in the special case where $Z_{\mathrm{t}}(\theta)$ is the state (vector of queur: lengths) in a closed Jackson-like queueing network. Through chrice of $f$ in (1), $\mathrm{E}[R(\theta)]$ could be, for example, the mean queue length at $t_{f}$ at a fixed node, or the probability that some node is idle at $t_{f}$.

We use the following notation:

$$
\begin{aligned}
\tau_{1} & =\text { epoch of } i t h \text { transition; } \\
Y_{1} & =\text { ith state }=Z_{\tau_{1}^{+}} \\
a_{2} & =\text { node from which ith departure occurs; } \\
\chi_{a} & =\text { quene joined by } j \text { th departure from node } a ; \\
X_{a j} & =j \text { th service time at } a \\
f_{a}, F_{a} & =\text { service time density and cdf at node } a .
\end{aligned}
$$

The service times $X_{a j}$ depend on $\theta$; the sequence, $\chi$, of routing indicators, and the initial state, $Y_{0}$, do not.

We first consider a (slightly rasier) discrete time version of terminal reward, given by $R_{n}(\theta)=f\left(Y_{n}(\theta)\right)$. We condition on

$$
\xi_{n}=\left(Y_{0} ; \tau_{1}, \ldots, \tau_{n} ; a_{1}, \ldots, a_{n+1} ; 入\right) .
$$

The node $a_{n+1}$ from which the nest departure occurs is included, but the epoch $\tau_{n+1}$ of its occurrence is not. Given $\xi_{n}$, we can reconstruct $Y_{1}, \ldots, Y_{n}$ and $\left\{Z_{t}, 0 \leq t \leq \tau_{n}\right\}$, but not the entire evolution of $Z_{t}$.

Let $a(\theta)=\left(a_{1}(\theta), \ldots, a_{n}(\theta)\right)$. Let $\mathcal{A}_{n}(\theta)$ be the set consisting of $a(\theta)$, any sequence that can be obtained from $a(\theta)$ by transposing some $a_{\imath}(\theta)$ and $a_{\imath+1}(\theta), i<n$, and also the sequence $a^{*}(\theta)=\left(a_{1}(\theta), \ldots, a_{n-1}(\theta), a_{n+1}(\theta)\right)$. If the service times satisfy (A1') and (A2') then

$$
P\left(a(\theta+h) \notin \mathcal{A}_{n}(\theta)\right)=o(h) .
$$

In-other words, the probabilty of multiple order changes among the service completions is negligible. Our estimator of the derivative of $\mathrm{E}\left[R_{n}(\theta)\right]$ will, therefore, take the form

$\sum_{a^{\prime} \in \mathcal{A}_{n}} Q\left(a^{\prime} ; \xi_{n}\right) \mathrm{E}\left[f\left(Y_{n}(\theta+h)\right)-f\left(Y_{n}(\theta)\right) \mid \xi_{n}, a(\theta+h)=a^{\prime}\right]$

where

$$
Q\left(a^{\prime} ; \xi_{n}\right)=\lim _{h\lfloor 0} \frac{1}{h} P\left(a(\theta+h)=a^{\prime} \mid \xi_{n}\right) .
$$

Given $\xi_{n}$ and $a(\theta+h), Y_{n}(\theta)$ and $Y_{n}(\theta+h)$ are known. Moreover, $Y_{n}$ does not depend on the order of $a_{1}, \ldots, a_{n}$. Given $x$ and $Y_{0}$, it is enough to know how many departures occurred from each node to determine $Y_{n}$; the order in which the departures occur does not change $Y_{n}$. If $a(\theta+h) \in \mathcal{A}_{n}(\theta)$ and $a(\theta+h) \neq a^{*}$, then $a(\theta+h)$ is just a permutation of $a(\theta)$ and $Y_{n}(\theta+h)=Y_{n}(\theta)$. Our estimator therefore reduces to

$$
Q\left(a^{*} ; \xi_{n}\right) \mathbf{E}\left[f\left(Y_{n}(\theta+h)\right)-f\left(Y_{n}(\theta)\right) \mid \xi_{n}, a(\theta+h)=a^{*}\right]
$$

If we define $Y_{n}^{*}$ to be the $n$th state when $a_{n}$ and $a_{n+1}$ change $\operatorname{order}\left(Y_{n}^{*} \in \sigma\left(\xi_{n}\right)\right)$, we get simply

$$
Q\left(a^{*} ; \dot{\zeta}_{n}\right)\left[\int\left(Y_{n}^{*}\right)-\int\left(Y_{n}\right)\right]
$$

It remains to calculate $Q\left(a^{*} ; \xi_{n}\right)$, which is the rate at which $a_{n}$ and $a_{n+1}$ change order. The general form of this rate should by now be clear:

$$
Q\left(a^{*} ; \xi_{n}\right)=\dot{f}(0)\left[\frac{d \tau_{n}}{d \theta}-\left(\frac{d \tau_{n+1}}{d \theta}\right)_{\tau_{n+1}=\tau_{n}}\right]^{+}
$$

$\hat{f}(0)$ is the conditional probability density that $a_{n+1}$ occurs just after $a_{n}$, and the $[\cdot]^{+}$term is the rate at which they change order, given that they orcur simultaneously. 
Given $\xi_{n}$, the residual time (at $\tau_{n}$ ) until $\tau_{n+1}$ is the residual service time at node $a_{n+1}$ conditioned on being the shortest residual service time at any node. If $t_{a}$ is the age (at $\tau_{n}$ ) of the current service time (if any) at node $a$, then this density is, for $x \geq 0$,

$$
\hat{f}(x) \sim f_{a_{n+1}}\left(t_{a_{n+1}}+x\right) \prod_{a}\left(1-F_{a}\left(t_{a}+x\right)\right),
$$

where the product runs over non-idle nodes $a$ other than $a_{n+1}$.

The second factor in $Q\left(a^{*} ; \xi_{n}\right)$ is evaluated using IPA. There is a sequence $X_{i_{k} J_{k}}$ (determined by $\xi_{n}$ ) such that

$$
\tau_{n}=\sum_{k} X_{\imath_{k} J_{k}}
$$

and

$$
\frac{d \tau_{n}}{d \theta}=\sum_{k} \frac{d X_{i_{k} \jmath_{k}}}{d \theta}=\sum_{k} D_{i_{k}}\left(X_{i_{k} \jmath_{k}}\right) .
$$

Similarly, if $\tau_{n+1}=\tau_{m}+X_{a_{n+1} k}-$ i.e., the service time that ends at $\tau_{n+1}$ began at. $\tau_{m}$ and is the $k t h$ service time at $a_{n+1}$ - then

$$
\left(\frac{d \tau_{n+1}}{d \theta}\right)_{\tau_{n+1}=\tau_{n}}=\frac{d \tau_{m}}{d \theta}+D_{a_{n+1}}\left(\tau_{n}-\tau_{m}\right) .
$$

Thus, these terms are easily computed as the simulation evolves.

Theorem 1. Suppose the service times satisfy (A1)-(A3) and have finite expectations. If $\mathbf{E}\left[R_{n}(\theta)\right]$ is differentiable, then

$$
\mathbf{E}\left[Q\left(a^{*} ; \xi_{n}\right)\left[f\left(Y_{n}^{*}\right)-f\left(Y_{n}\right)\right]\right]=\frac{d \mathbf{E}\left[R_{n}(\theta)\right]}{d \theta} .
$$

This (almost) follows from Theorem 1 of [7], which is a result about generalized semi-Markov processes. A condition less general than (A1) and (A3) is used in [7]; the gap is filled by the argument used to prove Proposition 1.

We now consider the case of $R(\theta)=f\left(Z_{t_{j}}(\theta)\right)$. Let $N_{t}$ be the number of transitions in $[0, t]$. Denote $N_{t_{f}}$ simply by $N$ and let $a=\left(a_{1}, \ldots, a_{N}\right)$. Let $\mathcal{A}$ include $a$ and sequences obtained through a single transposition, as before. But instead of $a^{*}$, add to $\mathcal{A}$

$$
a_{+}=\left(a_{1}, \ldots, a_{N}, a_{N+1}\right)
$$

and
The sequences $a_{+}$and $a_{-}$are, respectively, the result of order changes between $\tau_{N+1}$ and $t_{f}$, and between $\tau_{N}$ and $t_{f}$. If the service times satisfy (A1') and (A2),

$$
P(a(\theta+h) \notin \mathcal{A}(\theta))=o(h) .
$$

Moreover, of the elements of $\mathcal{A}(\theta)$, only $a_{+}$and $a_{-}$result in $Z_{t_{j}}(\theta+h) \neq Z_{t_{j}}(\theta)$, so we only need to consider these order changes. Let $Z_{+}$and $Z_{-}$denote $Z_{t_{f}}(\theta+h)$ when $a(\theta+h)$ is $a_{+}(\theta)$ and $a_{-}(\theta)$ respectively.

If we condition on $\xi_{+}=\xi_{N}$ and $\xi_{-}=\xi_{N-1}$, we get

$$
Q\left(a_{+} ; \xi_{+}\right)=\hat{f}_{+}(0)\left[-\left(\frac{d \tau_{N+1}}{d \theta}\right)_{\tau_{N+1}=t_{f}}\right]^{+}
$$

and

$$
Q\left(a_{-;} \xi_{-}\right)=\hat{f}_{-}(0)\left[\left(\frac{d \tau_{N}}{d \theta}\right)_{\tau_{N}=t_{f}}\right]^{+} .
$$

To evaluate the densities $\hat{f}_{+}$and $\hat{f}_{-}$, let $t_{a}$ be the age of the current service time at node $a$ at $t_{f}$. Then for all $x \geq 0$,

$$
\hat{f}_{+}(x) \sim f_{a_{N+1}}\left(t_{a_{N+1}}+x\right) \prod_{a}\left(1-F_{a}\left(t_{a}+x\right)\right),
$$

while for $0 \leq x \leq t_{f}-\tau_{N}$,

$$
\hat{f}_{-}(x) \sim f_{a_{N}}\left(t_{a_{N}}+x\right) \prod_{a}\left(1-F_{a}\left(t_{f}-\tau_{N}-x\right)\right) .
$$

In the first expression, the product runs over non-idle nodes other than $a_{N+1}$; in the second, it runs over those nodes (if any) that initiate a service time at $\tau_{N}$.

The following is similar to Theorem 3 of [7]:

Theorem 2. Under the conditions for Theorem 1,

$$
\begin{aligned}
\frac{d \mathrm{E}[R(\theta)]}{d \theta}= & \mathrm{E}\left[Q\left(a_{+} ; \xi_{+}\right)\left[f\left(Z_{+}\right)-f\left(Z_{t_{f}}\right)\right]\right. \\
& \left.+Q\left(a_{-} ; \xi_{-}\right)\left[f\left(Z_{-}\right)-f\left(Z_{t_{f}}\right)\right]\right] .
\end{aligned}
$$

\section{REMARKS ON SPA}

(5.1). The SPA estimator calculated above for the derivative of expected terminal reward in queueing networks extends to a class of generalized semi-Markov processes (GSMPs). If an denotes the nth event to occur, and $Y_{n}$ is the nth state visited by the GSMP, the main condition we require is that $Y_{n}$ not depend on the order of $a_{1}, \ldots, a_{n}$. This also happens to be an important condition in applying IPA to cumulative functionals (like (2)) of GSMPs. 
(5.2). The function $\Phi_{\imath}$ required by (A1) never appears in the SPA estimator and need not be known explicitly. All that is needed is $D_{1}-$ the derivative of $\Phi_{1}$ at $h=0$. As noted above, a candidate $D_{2}$ is provided by (11).

(5.3). Evaluation of the densities $\hat{f}(0)$ that enter into the jump rates $Q(\cdot ; \cdot)$ can be difficult. (A notable exception is the case where all $X_{2}$ are exponential.) In general, conditioning on more information makes calculation of $Q$ easier; but conditioning on too much may fail to smooth jumps. Hence, SPA tentatively prescribes

Condition on the largest $\mathcal{F}$ that makes $\mathbf{E}[\gamma(\theta+h)-\gamma(\theta) \mid \mathcal{F}]$ continuous in $h$.

On the other hand, in considering variance, the principle of conditional Monte Carlo (Fox and Glynn [3], Proposition 1) applies: If $\mathcal{F} \subseteq \mathcal{G}$, then for all $h$,

$$
\mathrm{E}\left[\mathrm{E}\left[\frac{\gamma(\theta+h)-\gamma(\theta)}{h} \mid \mathcal{F}\right]^{2}\right] \leq \mathrm{E}\left[\mathrm{E}\left[\frac{\gamma(\theta+h)-\gamma(\theta)}{h} \mid \mathcal{G}\right]^{2}\right] .
$$

Modulo an additional interchange of limit and expectation, this implies that an unbiased SPA estimator based on $\mathcal{F}$ has smaller variance than one based on $\mathcal{G}$. Hence, variance reduction suggests

Condition on the smallest $\mathcal{F}$ that makes $\mathbf{E}[\gamma(\theta) \mid \mathcal{F}]^{\prime}$ tractable.

In practice, the first of these considerations seems to be the more critical one.

\section{TERMINAL REWARD VIA DYNKIN'S FORMULA}

We now describe a different method for estimating the sensitivity of expected terminal reward. Let $Z_{t}$ be a Markov process with infinitesimal generator $\mathbf{Q}$. Dynkin's formula states that if $T$ is a stopping time (for $Z_{t}$ ) with finite expectation,

$$
\mathrm{E}\left[f\left(Z_{T}\right)\right]=\mathrm{E}\left[f\left(Z_{0}\right)\right]+\mathrm{E}\left[\int_{0}^{T} \mathrm{Q} f\left(Z_{t}\right) d t\right] .
$$

(See, for example, Karlin and Taylor [10], p.298.) Since $\mathbf{Q}$ can be defined by

$$
\mathbf{Q} f(x)=\lim _{h \downarrow 0} \frac{\mathrm{E}\left[f\left(Z_{h}\right) \mid Z_{0}=x\right]-f(x)}{h},
$$

(24) is reminiscent of the fundamental theorem of calculus. Taking $T=\tau_{n}$ and $T=t_{f}$ covers the two cases considered in Section 4 .

Suppose, now, that $\mathbf{Q}$ (hence, $Z_{t}$ ) depends on a parameter $\theta$. If $Z_{0}$ is fixed, then $\partial_{\theta} \mathbf{E}\left[f\left(Z_{0}\right)\right]=0$. Thus, in this case, Dynkin's formula transforms a terminal reward problem to a cumulative reward problem with which we can try to use IPA. The problem we end up with does not quite have the form (2). Instear, we must consider

$$
\int_{0}^{t_{f}} g\left(\theta, Z_{t}(\theta)\right) d t
$$

where

$$
g(\theta, x)=\mathbf{Q}_{\theta} f(x)=\sum_{y} \mathbf{I}_{\theta}(x, y) f(y)
$$

depends explicitly on $\theta$ because $\mathbf{Q}_{\theta}$ does.

Consider the case of (right-continuous, non-explosive) $Z_{t}(\theta)$ on a discrete state space. Let $Y_{t}(\theta)$ be the ith state visited by $Z_{t}(\theta)$; let $\tau_{t}(\theta)$ be the epoch of the ith transition of $Z_{t}(\theta)$; and let $N=N_{t_{f}}(\theta)$ be the number of transitions in $\left[0, t_{f}\right]$. We may rewrite $(25)$ as

$$
\sum_{i=0}^{N-1} g\left(\theta, Y_{i}(\theta)\right)\left[r_{i}+1-\tau_{i}\right]+\left(T-\tau_{N}\right) g\left(\theta, Y_{N}(\theta)\right) .
$$

Thus, differentiating (25), we get

$$
\int_{0}^{t_{f}} \partial_{\theta} g\left(\theta, Z_{t}(\theta)\right) d t+\sum_{i=1}^{N} \frac{d \tau_{i}}{d \theta}\left[g\left(\theta, Y_{i-1}\right)-g\left(\theta, Y_{i}\right)\right] .
$$

(In this expression, the int egrand is the partial derivative of $g$ with respect to its first argument only; that is, with respect to the explicit dependence on $\theta$.) Sufficient conditions for the IPA estimate (26) to be unbiased are given in [6]. Other conditions on $Z_{t}$ - based on viewing it as a GSNP with exponential clock times - can be based on the ronditions in [5]; cf. Remark 5.1.

This approach to sensitivity analysis of expected terminal reward can be applied even when $Z_{t}$ is not naturally Marko vian; or, to put it another way, when supplementary variables must be appended to make $Z_{t}$ a Markov process. Burman [1], p.855, exhibits the infinitesimal generator for GSMPs. In [1], reward functions $f$ that depend on clock ages are allowed; but here we restrict attention to functions that depend only on the discrete part of the state. Using $s, s^{\prime}$ to denote states, $\alpha$ to denote events, $p\left(s^{\prime} ; s, \alpha\right)$ for transition probabilities, $t_{\alpha}$ to denote the age of the a-clock and $\mu_{\alpha}\left(t_{\alpha}\right)$ for the hazard rate of the a-clock, we get

$$
\mathrm{Q} f\left(s,\left\{t_{\alpha}\right\}\right)=\sum_{\alpha} \sum_{s^{\prime}} p\left(s^{\prime} ; s, a\right) \mu_{\alpha}\left(t_{\alpha}\right)\left[f\left(s^{\prime}\right)-f(s)\right] .
$$

(Burman [1] allows speeds.) If only the clock distributions depend on $\theta$, this yields the IPA intimate

$$
\int_{0}^{t_{f}} \theta_{\theta} \mathbf{Q}_{\theta}\left(Z_{t}\right) d t+\sum_{i=1}^{N} \frac{d \tau_{1}}{d \theta}\left[\mathbf{Q}_{\theta} f\left(Z_{r_{1}}\right)-\mathbf{Q}_{\theta} f\left(Z_{\tau_{i}-}\right)\right]
$$

where 


$$
\begin{aligned}
& \partial_{\theta} \mathbf{Q}_{\theta} f\left(s,\left\{t_{\alpha}\right\}\right)= \\
& \quad \sum_{\alpha, s^{\prime}}\left(\frac{\partial \mu_{\alpha}\left(t_{\alpha}\right)}{\partial \theta}+\frac{\partial \mu_{\alpha}\left(t_{\alpha}\right)}{\partial t_{\alpha}} \frac{d t_{\alpha}}{d \theta}\right) p\left(s^{\prime} ; s,(\alpha)\left[f\left(s^{\prime}\right)-f(s)\right] .\right.
\end{aligned}
$$

\section{FURTHER REMARKS}

(7.1). It should be stressed that, perhaps surprisingly, the domains of SPA estimaters for terminal reward, and estimators based on Dynkin's formula plus IPA are roughly the same. The main condition that makes IPA work (see Remark 5.1) also makes SPA feasible. The significance of this condition is that it "localizes" the effect of event order changes, which is important for both methods. The relative efficiency of the two methods is problem-dependent.

(7.2) In SPA, conditioning is used to smooth discontinuities due to changes in $\theta$. In Dynkin's formula, conditioning smooths the dependence on $t$, via the definition of $\mathbf{Q}$.

(7.3) Let $A_{t}$ be a point process and $\lambda_{t}$ an intensity for $A_{t}$ Zazanis [11] considers derivative estimators that smooth by replacing an integral against $d A_{t}$ with one against $\lambda_{t} d t$, both integrals having the same expectation. Since

$$
f\left(Z_{T}\right)-f\left(Z_{0}\right)=\int_{0}^{T} d f\left(Z_{t}\right),
$$

Dynkin's formula (24) can be viewed as replacing an integral against $d f\left(Z_{t}\right)$ with one against $\mathbf{Q} f\left(Z_{t}\right) d t=\mathrm{E}\left[d f\left(Z_{t}\right) \mid Z_{t}\right]$, where, again, both have the same expectation.

(7.4) There is a class of problems, which we now describe briefly, for which Dynkin's formula and SPA yield the same estimator. Consider, again, the case of a discrete state Markov process. Call $\theta$ a scale parameter if whenever $\mathbf{q}_{\theta}(x, y)>0, \mathbf{q}_{\theta}^{\prime}(x, y) / \mathbf{q}_{\theta}(x, y)=\mathbf{q}_{\theta}^{\prime}(x) / \mathbf{q}_{\theta}(x)$. This makes $\left[\mathbf{1}_{\theta}(x, y) / \mathbf{q}_{\theta}(x)\right]^{\prime}=0$, so $\theta$ is a parameter of the holding times but not the embedded transition probabilities. Suppose $\theta$ is a scale parameter and suppose also that every $\mathbf{q}_{\theta}^{\prime}(x) \geq 0$ (i.e., the holding times are decreasing in $\theta$ ). Then the IPA estimator obtained via Dynkin's formula coincides with the SPA estimator for terminal reward based on

$$
\xi=\left(Y_{0}, \ldots, Y_{N} ; \tau_{1}, \ldots, \tau_{N}\right)
$$

where $N \equiv N_{t}$ is the number of transitions in $\left[0, t_{f}\right]$. Though we omit the derivation, both estimators become

$$
\mathrm{Q} f\left(Z_{t_{\jmath}}\right) \cdot \int_{0}^{t_{f}} \frac{\mathrm{q}^{\prime}\left(Z_{t}\right)}{\mathrm{q}\left(Z_{t}\right)} d t .
$$

(7.5). Our results for expreted terminal reward have implirations for steady-state derivative estimation. If, say, $Z_{t}(\theta) \Rightarrow Z_{\infty}(\theta)$ and

$$
\lim _{t \rightarrow \infty} \frac{d \mathbf{E}\left[f\left(Z_{t}(\theta)\right)\right]}{d \theta}=\frac{d \mathbf{E}\left[f\left(Z_{\infty}(\theta)\right)\right]}{d \theta}
$$

then our estimators of $d \mathbf{E}\left[f\left(Z_{t}(\theta)\right)\right] / d \theta$ are asymptotically unbiased estimators of the derivative of the steady-state mean $\mathbf{E}[f(Z \ldots(0))]$.

(7.6). In estimaling the derivative of expected terminal reward via likelihood ratios, the fact that $R$ is discontinuous is irrelevant. Even so, Dynkin's formula may have applications here as well. If $L_{t_{f}}(\theta)$ is the likelihood of $\left\{Z_{t}, 0 \leq t \leq t_{f}\right\}$ under $\mathbf{Q}_{\theta}$, then

$$
\int_{0}^{t_{f}} \mathbf{Q}_{\theta} f\left(Z_{t}\right) d t \cdot \frac{L_{t_{j}}^{\prime}(\theta)}{L_{t_{f}}(\theta)}
$$

may have smaller variance than

$$
f\left(Z_{t_{f}}\right) \cdot \frac{L_{t_{f}}^{\prime}(\theta)}{L_{t_{f}}(\theta)} .
$$

Fox [2] reports the same order-of-magnitude computational costs for estimating cumulative reward and its derivative as for terminal reward and its derivative, so the transformation is worth considering.

\section{REFERENCES}

[1] Burman, D.Y., "Insensitivity in queueing systems", $A d$ vances in Applied Probability, 13, pp.846-859, 1981.

[2] Fox, B.L., "Complexity of gradient estimation for transient Markov chains", Technical Report, University of Montreal, 1987.

[3] Fox, B.L. and Glynn, P.W., "Discrete time conversion for simulating finite-horizon Markov processes", Technical Report, University of Colorado, Denver, 1989.

[4] Glasserman, P., "Performance continuity and differentiability in Monte Carlo optimization", Proceedings of the Winter Simulation Cionference, M. Abrams, P. Haigh and J. Comfort, (eds.), pp.518-524, 1988.

[5] Glasserman, P., "Structural condtions for perturbation analysis derivative estimation: finite-time performance indices", submitted for publication, 1988.

[6] Cilasserman, P., "Derivative estimates from simulation of continurus-time Markov chains", submitted for publication, 1989 .

[7] Glasserman, P. and Gong, W.B., "Smoothed perturbation analysis for a class of discrete event systems", submitted for publication, 1989.

[8] Gong, W.B. and Ho, Y.C., "Smoothed perturbation analysis of discrete event dynamical systems", IEEE Transactions on Automatic Control, 32, pp.858-866, 1987. 
[9] Heidelberger, P. and Goyal, A., "Sensitivity analysis of continuous time Markov chains using unifornization", in 2nd International Workshop on Applied Mathematics and Performance/Reliability Models of Ciomputer/Communication Systems, University of Rome II, pp.93-104, 1987.

[10] Karlin, S. and Taylor, H.M., A Second Course in Stochastic Processes, Academic Press, New Y'ork, 1981.

[11] Zazanis, M., "Compensators and Derivative Estimation for Queueing Systems", Procfedings of the 26th Allerton Conference, pp.549-555, 1988.

PAUL GLASSERMAN is Member of Technical Staff in the Operations Research Department at AT\&T Bell Laboratories. He received a Ph.D. from the Division of Applied Science, Harvard University in 1988, and an A.B. in Mathematics from Princeton University in 1984. His interests include derivative estimation, Monte Carlo optimization and queueing theory.

Room 3K-324

AT\&:T Bell Laboratories

Crawfords Corner Rd.

Holmdel, NJ 07733

WEI-BO GONG received the M.S. degree in engineering from the China University of Sciences and Technology in 1981. He worked as an Instructor at the same university for two years. In 1987 he received the Ph.D. degree from Harvard Iniversity. He is on the faculty of the Department of Electrical and Computer Engineering, University of Massachusetts at Amherst. His research interests include analysis and optimization of discrete event systems, such as computercommunication net works and flexible manufacturing systems.

Electrical and Computer Engincering

Eniversity of Massachusetts, Amherst

Amherst, MA 01003 